Observat i on of Hydrogen and Cesi um Spectra in a Negat i ve I on Source for a Neut $r$ al Beam Inj ect or usi ng a Mul i-channel Spect rometer

\begin{tabular}{|l|l|}
\hline $\begin{array}{l}\text { jour nal or } \\
\text { publ i cat i on ti t l e }\end{array}$ & Pl asma and Fusi on Research \\
\hline vol une & Vol . 2 \\
\hline page range & S1047- 1 - S1047- 4 \\
\hline year & 2007- 01- 01 \\
\hline URL & ht t p: //hdl . handl e. net /10655/6334 \\
\hline
\end{tabular}




\title{
Observation of Hydrogen and Cesium Spectra in a Negative Ion Source for a Neutral Beam Injector using a Multi-Channel Spectrometer
}

\author{
K. IKEDA, U. FANTZ1) ${ }^{1}$, K. NAGAOKA, Y. TAKEIRI, M. OSAKABE, K. TSUMORI, \\ O. KANEKO and Y. OKA \\ National Institute for Fusion Science, Toki, Gifu 509-5292, Japan \\ 1) Max-Planck-Institut fuer Plasmaphysik, Boltzmannstrasse 2 D-85748, Garching, Germany
}

(Received 4 December 2006 / Accepted 2 April 2007)

\begin{abstract}
Effective production of hydrogen negative ions $\left(\mathrm{H}^{-}\right)$is one of the important issues in high power neutral beam injection (NBI) for fusion experimental devices. Cesium (Cs) vapor is seeded in the $\mathrm{H}^{-}$sources to enhance the $\mathrm{H}^{-}$production rate, which depends strongly on the amount of $\mathrm{Cs}$ inside the source. On the other hand, oxygen and water attenuate the production rate. In order to investigate the states of those species inside ion source, we have installed two multi-channel spectrometer systems with different spectral resolution. Hydrogen emissions, Cs emissions and oxygen emission are clearly observed with the time resolution of several handled milliseconds. Most of evaporated Cs atoms ionize in arc discharge plasma, and the emission increases strongly due to the ion back streaming with beam extraction. Oxygen neutral emission increases at the beginning of the beam conditioning, and its evaporation depends on the condition of the grid system.
\end{abstract}

(c) 2007 The Japan Society of Plasma Science and Nuclear Fusion Research

Keywords: negative ion source, spectroscopy, hydrogen, cesium, oxygen

DOI: $10.1585 /$ prr.2.S1047

\section{Introduction}

High-energy neutral beam injection (NBI) system used a negative ion source is a key device to heat a plasma for fusion research. Hydrogen negative ion $\left(\mathrm{H}^{-}\right)$source used cesium (Cs) vapor, which is effective to improve $\mathrm{H}^{-}$ current density, have been developed in many research facilities [1-3]. We have adopted six large $\mathrm{H}^{-}$sources used arc discharge for the Large Helical Device (LHD) experiment, and these are successfully operated. Injection beam power has been reached $13 \mathrm{MW}$ totally three beam lines [4]. The current density of $\mathrm{H}^{-}$at the exit of ion source is achieved $350 \mathrm{~A} / \mathrm{m}^{2}$ [5]. So, the information of the plasma parameters and $\mathrm{Cs}$ behavior in our $\mathrm{H}^{-}$source contribute for the development of the future large negative ion source such as the ITER-NBI system.

It is important to obtain the behavior of Cs and impurities in the $\mathrm{H}^{-}$sources for improving $\mathrm{H}^{-}$production efficiency and beam uniformity. Recently, the relationship of plasma parameters and the production of the negative hydrogen ions are researched well by using an optical emission spectroscopy technique [6]. Optical emission provides the information of its particle density and temperature used collisional radiative models [7].

We have adopted an optical emission spectroscopy diagnostic in order to investigate the state of Cs and impurities inside a large $\mathrm{H}^{-}$source. We will show the history of

author'se-mail: ikeda.katsunori@lhd.nifs.ac.jp the Cs signals and oxygen impurity signals at the beginning of the beam conditioning with a different condition of the grid systems.

\section{Optical Design on Ion Source}

Figure 1 shows a structure of a $\mathrm{H}^{-}$source and measurement devices. Hydrogen neutral beam with the energy of $180 \mathrm{keV}$ is extracted by five segment of the grid system, which consists of the plasma grids (PG), extraction grids (EG), and grounded grids. Hydrogen plasma is produced by an arc discharge used the twenty-four hairpin-shaped tungsten filaments installed at the side plate of the chamber wall. The inner size of the arc chamber are $1450 \mathrm{~mm}$ high, $350 \mathrm{~mm}$ wide, and $223 \mathrm{~mm}$ depth from the back plate surface to the PG. We use twelve units of filament and arc power supplies to control an arc discharge distribution [8]. Three Cs ovens are equipped on the back plate of the arc chamber to seed Cs vapor. Two Langmuir probes are installed at the port \#6 $(y=87 \mathrm{~mm})$ and $\# 7(y=-435 \mathrm{~mm})$ on the left side $(x=-70 \mathrm{~mm})$ of the back plate to measure an electron temperature and an electron density of the arc discharge plasma without beam extraction. These probes are made by tungsten wire with the diameter of $1 \mathrm{~mm}$ covered a ceramic tube, and it can be moved along the $z$ direction.

We arrange the vertical sight line used the optical view port $\# 1(z=106 \mathrm{~mm})$ on the top of the arc chamber. And also, four grid sight lines used the optical view ports \#2 $(y=435 \mathrm{~mm}), \# 3(y=87 \mathrm{~mm}), \# 4(y=-87 \mathrm{~mm})$, 
and $\# 5(y=-435 \mathrm{~mm})$ are arranged on the right side $(x=70 \mathrm{~mm})$ of the back plate. Plasma emission is focused on quartz optical fibers with $0.23 \mathrm{~mm}$ clad core diameter by achromatic lenses after through a quartz window and a neutral density filter. The end of optical fiber faced on the plasma is arranged a FC type optical connector within four fiber cores, and the other end of the fiber coupled with the spectrometer is split into two FC type and two SMA905 type optical connectors as shown in Fig. 1.

We have arranged the multi-channel survey spectrometers as shown in Fig. 2. Four wide range survey spectrometers (Plasus, EmiCon $4 \mathrm{ch}$ ) take many spectra simultaneously from the $200 \mathrm{~nm}$ to $870 \mathrm{~nm}$ with a low-resolution of $1.5 \mathrm{~nm}$ with typically $200 \mathrm{~ms}$ interval of time. A high-

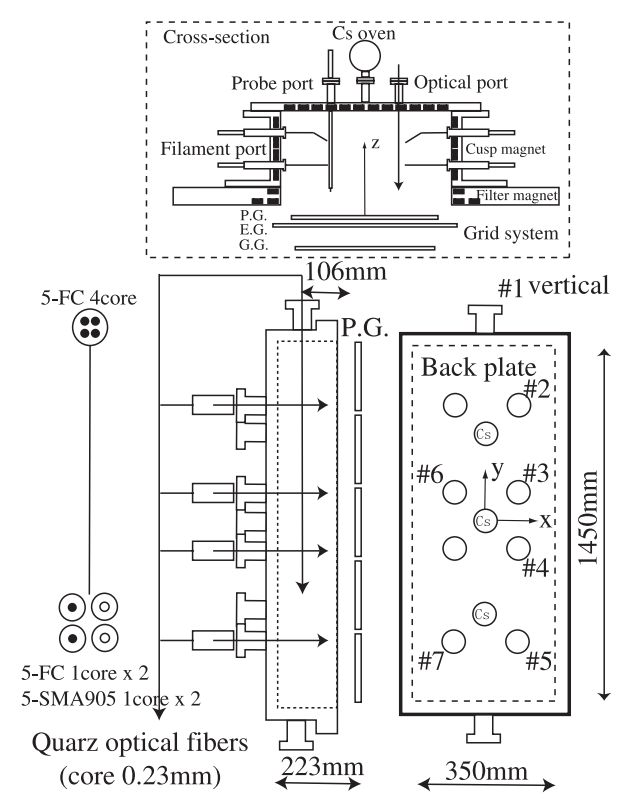

Fig. 1 Schematic view of the $\mathrm{H}^{-}$source for NBI in LHD. Three Cs ovens, two movable Langmuir probes and four optical sight lines are installed on the back plate, and the vertical sight line is installed on the top plate of the ion source.

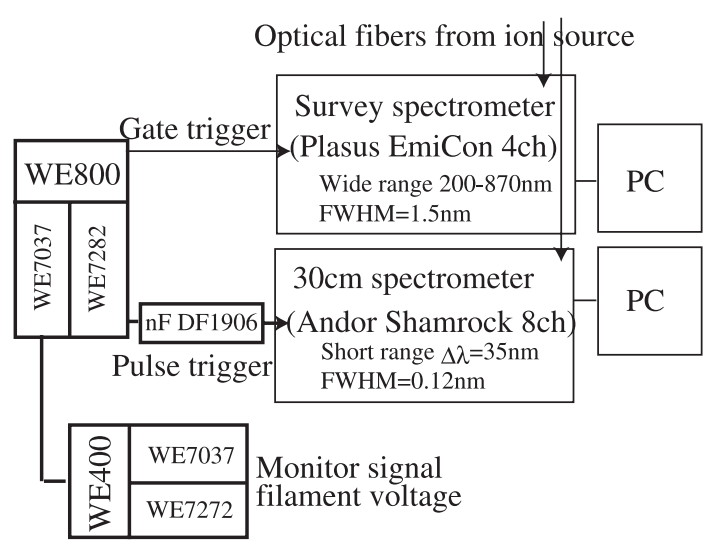

Fig. 2 Four wide range ( $200 \mathrm{~nm} \sim 870 \mathrm{~nm})$ survey spectrometers and high-resolution $(\mathrm{FWHM}=0.12 \mathrm{~nm}) 8$ channels spectrometer are connected at the end of quartz optical fibers. resolution $(0.12 \mathrm{~nm})$ spectrometer (Andor, Shamrock $8 \mathrm{ch}$ ) used 1800 grooves/mm holographic grating takes closeup spectra into the $35 \mathrm{~nm}$ narrow wavelength range with $500 \mathrm{~ms}$ interval of time. A CCD image sensor, with the size of 1024 pixels width and 256 pixels height, is cooled to -60 degrees to reduce the thermal noise. These spectrometer systems included the optical configurations are absolutely calibrated by the standard lamp with Urbricht sphere (SphereOptics, LR-12-M) with the same distance of the experimental configuration.

\section{Results}

\subsection{Langmuir probe}

Basic plasma parameters of both an electron density and an electron temperature are measured by the Langmuir probes as shown in Fig. 3. We use $80 \mathrm{~kW}$ arc power discharge with $\mathrm{Cs}$ vapor in the condition of $0.3 \mathrm{~Pa}$ hydrogen gas pressure and $3 \mathrm{~V}$ bias voltage between the PG and arc chamber. The electron density increases to $n_{e}=3 \times 10^{18} \mathrm{~m}^{-3}$ around the center of the arc chamber $(z=120 \mathrm{~mm})$ near the filament position $(z=106 \mathrm{~mm})$, where is the discharge aria of hydrogen plasma. The electron temperature is $4 \mathrm{eV}$ around the same discharge area. The electron density and electron temperature decrease near the PG $(z<50 \mathrm{~mm})$, where exists the strong magnetic filter field. The focal point of grid optic is set to the point of $z=27 \mathrm{~mm}$ where is the $1 \mathrm{eV}$ electron temperature, and thin electron density.

\subsection{Emission spectra in arc discharge plasma}

Figure 4 shows the typical spectrum of the arc discharge plasma with $\mathrm{Cs}$ vapor. We have identified neutral hydrogen Balmer emissions $(\mathrm{H} \alpha, \mathrm{H} \beta, \mathrm{H} \gamma, \mathrm{H} \delta), \mathrm{Cs}^{+}$ emissions $(460 \mathrm{~nm}, 522 \mathrm{~nm})$, small neutral $\mathrm{Cs}^{0}$ emission $(852 \mathrm{~nm})$, small neutral oxygen $\mathrm{O}^{0}$ emission $(777 \mathrm{~nm})$ and molecular hydrogen emissions $(600 \sim 640 \mathrm{~nm})$ by using the low-resolution survey spectrometer as shown in Fig. 4 (a). These emission lines ride on strong continuum from the

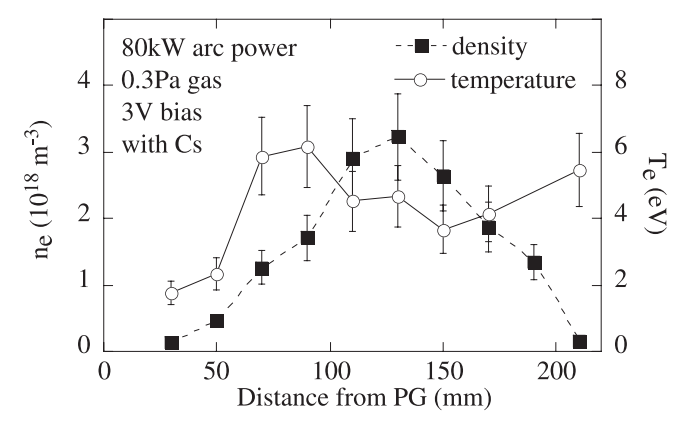

Fig. 3 Spatial profile of the electron density (square marks) and the electron temperature (circle marks) used a Langmuir probe in the $80 \mathrm{~kW}$ arc power discharge with $0.3 \mathrm{~Pa}$ gas pressure and $3 \mathrm{~V}$ bias voltage. 

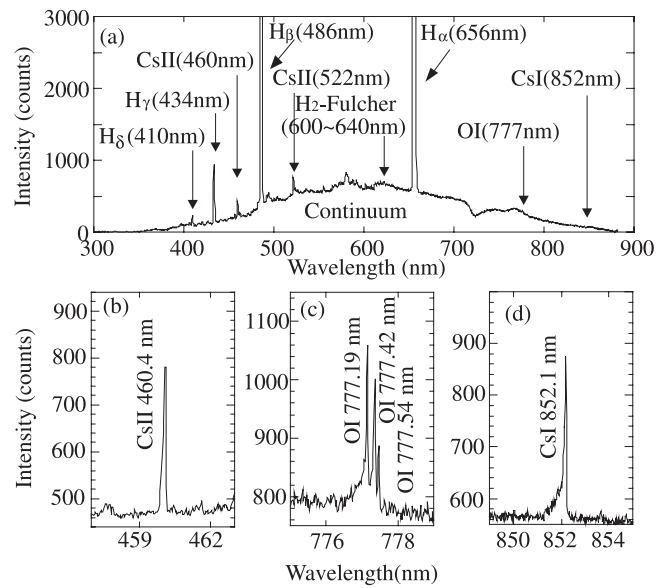

Fig. 4 Spectra of hydrogen Balmer emissions ( $\mathrm{H} \alpha, \mathrm{H} \beta, \mathrm{H} \gamma$ and $\mathrm{H} \delta$ ), $\mathrm{H}_{2}$ emissions, Cs emissions and oxygen emissions are observed by the wide range survey spectrometer (a). Detail structure of the Cs ion line (b), Cs neutral line (d) and oxygen neutral lines (c) are also checked by the highresolution spectrometer.

tungsten filaments for arc discharge. A time trace of the spectrum intensity is taken as the integral value of each spectrum removed continuum background. The structure of the $\mathrm{Cs}^{+}$line, the small $\mathrm{O}^{0}$ line and $\mathrm{Cs}^{0}$ line are also identified by high-resolution spectrometer as shown in Fig.4(b), (c) and (d), respectively. Time trace of the oxygen signal integrates three lines $(777.19 \mathrm{~nm}, 777.42 \mathrm{~nm}$, $777.54 \mathrm{~nm}$ ), and the other large impurity emissions are not observed around these spectra. We also estimate the hydrogen gas temperature as $2200 \mathrm{~K} \pm 300 \mathrm{~K}$ by the rotational structure of the hydrogen molecular emissions [9], and typical $\mathrm{H} / \mathrm{H}_{2}$ ratio is estimated as 0.35 from the ratio of the $\mathrm{H} \gamma$ emission and $\mathrm{H}_{2}$ emissions. We also check the neutral tungsten line (WI $400.9 \mathrm{~nm}$ ) from the evaporation of the tungsten filament, but it is not observed in our ion source.

Figure 5 (a) shows a time trace of a typical arc discharge with beam extraction. Arc power is $135 \mathrm{~kW}$ after the $10 \mathrm{sec}$ since turning on the filament power supply. The $136 \mathrm{kV}$ acceleration voltage is applied from $t=13 \mathrm{~s}$ to $t=15 \mathrm{~s}$, and the arc power supplies turn off at $3 \mathrm{sec}-$ ond after the beam extraction. $\mathrm{H} \gamma$ emission corresponds to the arc discharge, and the signal from the vertical sight (port \#1) is larger than the grid sight (port \#4) as shown in Fig. 5 (b), because the vertical sight views high density area, here the integral length of $1.4 \mathrm{~m}$ at the vertical sight and $0.2 \mathrm{~m}$ at the center grid sight are taken into account. On the other hand, $\mathrm{Cs}^{0}$ and $\mathrm{Cs}^{+}$emissions are rapidly increase by the beam extraction as shown in Fig. 5 (c) and (d). The additional Cs evaporates from the surface of the back plate of the arc chamber due to the impact of back streaming $\mathrm{H}^{+}$, those behaviors are consistent for the previous our experiment [10]. We also observe the Cs evaporation increase by increasing of the beam energy and the beam duration, then the Cs emission after the beam is

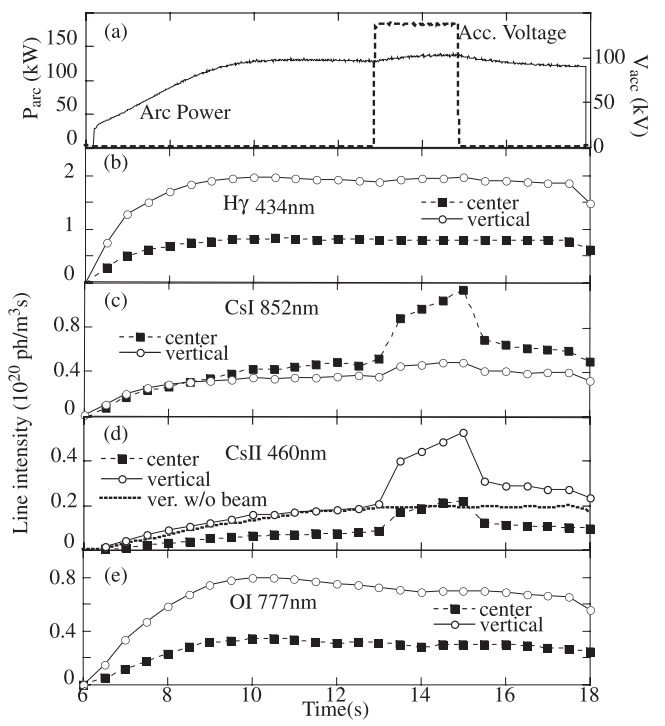

Fig. 5 Time evolutions of the $\mathrm{H} \gamma$ emission (b), $\mathrm{Cs}^{0}$ emission (c), $\mathrm{Cs}^{+}$emission (d) and $\mathrm{O}^{0}$ emissions (e) in the typical $135 \mathrm{~kW}$ arc discharge with beam extraction (a).

larger than that before the beam extraction. It is a weak $\mathrm{Cs}^{0}$ emission at the vertical sight, since the electron temperature along the vertical sight is larger than the $3.89 \mathrm{eV}$ Cs ionization potential. Here, we can estimate the ratio of $\mathrm{Cs}^{+} / \mathrm{Cs}^{0}$ as 0.99 at the vertical position from the emission coefficient at $\mathrm{T}_{\mathrm{e}}=4 \mathrm{eV}$. Increasing rate of $\mathrm{Cs}^{0}$ emissions at beam on time in the vertical sight is much smaller than that in the grid sight. So, the evaporated Cs form the back plate is also ionized around the vertical sight. We have not observed additional increasing of the $\mathrm{O}^{0}$ emissions in the beam extraction as shown in Fig. 5 (e). Oxygen signal decreases shot by shot, and day-by-day, and its density is estimated as $1.5 \times 10^{17} \mathrm{~m}^{-3}$, which corresponds to $3 \%$ of the electron density used the emission rate coefficient at the electron density of $4.5 \times 10^{18} \mathrm{~m}^{-3}$ obtained from the hydrogen emission ratio $\mathrm{H} \beta / \mathrm{H} \gamma=6.5$.

\subsection{History of the beam conditioning}

Figure 6 shows the history of the beam conditioning on two ion sources of IS3A and IS3B. Figure 6 (a) shows an arc discharge power and acceleration drain current. Figure 6 (b) shows the Cs history and the beam profile width on the beam calorimeter. Figure $6(\mathrm{c})$ and $6(\mathrm{~d})$ are the oxygen density and ratio of the oxygen and electron density estimated from the optical measurement, respectively. The IS3A is the rebuild ion source after the maintenance, and its grid systems are exposed to air condition in seven month. The IS3B is the operated ion source in one month before opening the ion source for changing filaments and wiping inner wall by water, and it is exposed to air condition in only one day.

Two ion sources are operated without Cs vapor in the beginning two days with the low power arc dis- 


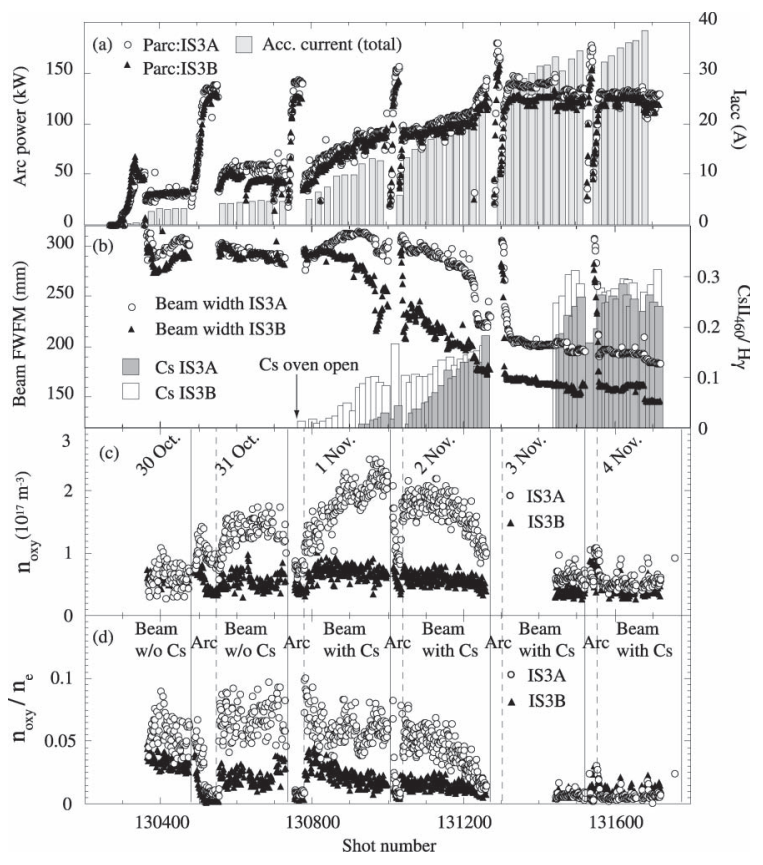

Fig. 6 History of the beam conditioning of two ion sources in the first week. Acceleration drain current increases as increasing arc discharge power with the Cs vapor (a). Cs signal increases after opening Cs ovens, and also the beam width of IS3B decreases quickly with a peaked beam distribution (b). Oxygen impurity densities and the density ratio $n_{\text {oxy }} / n_{e}$ estimated by the emission intensity are shown in (c) and (d), respectively.

charge $(\sim 50 \mathrm{~kW})$ and low acceleration voltage around $100 \mathrm{kV}$ as shown in Fig. 6 (a). Discharge of high arc power $(\sim 150 \mathrm{~kW})$ becomes possible in the second day, and the oxygen ratio decreases at only arc discharges. We have observed a large oxygen signal in the rebuild ion source IS3A with a low power beam extraction, and also it has a lot of electric break down at the grid systems in two days. Beam width does not change on the both ion source, as shown in Fig. 6 (b), because of a flat beam profile with low peak in the pure volume operation.

After the Cs seeding with same oven temperatures, it has a different behavior of oxygen signal in IS3A and IS3B as shown in Fig. 6 (c). It has small oxygen increasing into the IS3B, but we have observed the large increasing of the oxygen impurity into the IS3A. If the cause of large oxygen increasing is vacuum leak around a Cs oven, oxygen signal increases immediately, and it might not be saturated. We have checked a vacuum leak both the ion source and $\mathrm{Cs}$ ovens with opening oven valve before operating the ion source. If oxygen impurities come from an instrument downstream of the ion source, oxygen increasing must be appeared in both IS3A and IS3B. Different increasing of the oxygen appears in beam extraction, and we use the grid systems with different histories. So oxygen impurities seem to sputter from the IS3A grid system due to the in- creasing of the heat loading of the $\mathrm{H}^{-}$beam. Beam width of IS3B immediately decreases with a peaked beam profile, and we have observed increasing of the Cs signal and at the end of the third day. However, we have not observed a decreasing of the beam width with a flat beam profile without large increasing of the Cs signal in IS3A. Growth of the $\mathrm{H}^{-}$beam is slow to the same cesium feeding. In the fourth day, we find a lot of oxygen at the morning arc discharge without beam extraction in IS3A, because it sputters from the wall surface with Cs adsorbed oxygen. Oxygen sputtering signals decrease shot by shot, and oxygen ratio is also decreased in IS3A as shown in Fig. 6 (c) and (d). Then we have clearly observed the decreasing beam width with the peaked beam profile and rapidly increasing of Cs signals at the same time as shown in Fig. 6(b). This behavior corresponds to the decreasing of oxygen signals. Growth of the $\mathrm{H}^{-}$beam production may be obstructed by oxygen impurities adsorbing the surface $C$ s on plasma grids in the beginning of the beam conditioning. Period of the beam conditioning for the negative ion source becomes several days slow, but it does not become the serious delay

In conclusion, we applied the optical measurement systems in order to investigate the Cs and impurity behavior in $\mathrm{H}^{-}$ion source. Hydrogen, $\mathrm{Cs}$ and oxygen emissions are clearly observed. We found that oxygen impurity, which affects the production of $\mathrm{H}^{-}$, depends on the initial condition of the ion source and grid systems.

\section{Acknowledgments}

The authors would like to thanks the technical staff for their effort to support the NBI operation. This work is supported by NIFS06ULBB501. And also, this work is supported by the program of Japan Society for the Promotion of Science Bilateral Joint Projects and Seminars 2006.

[1] Y. Takeiri et al., Rev. Sci. Instrum. 71, 1225 (2006).

[2] E.Speth et al., Nucl. Fusion 46, S220 (2006).

[3] M. Kuriyama et al., J. of Nucl. Science and Technol. 35, 739 (1998).

[4] Y. Takeiri et al., "Confinement Improvement in High-Ion temperature Plasmas Heated with High Energy Negative NBI in LHD”, Fusion Energy 2006 (Proc. 21 th IAEA Int, Conf., Chengdu, 2006) EX/P4-42 (2006).

[5] O. Kaneko et al., "High Performance Operation of Negative-Ion-Based Neutral Beam Injection System for the Large Helical Device", Fusion Energy 2006 (Proc. 21th IAEA Int, Conf., Chengdu, 2006), FT/P5-4 (2006).

[6] U. Fantz, H.D. Falter, P. Franzen, and E. Speth, Rev. Sci. Instrum. 77, 03A516 (2006).

[7] U. Fantz, H.D. Falter, P. Franzen et al., Nucl. Fusion 46, S297 (2006).

[8] K. Ikeda, Y. Takeiri et al., Rev. of Sci. Instrum. 75, 1744 (2004).

[9] U. Fantz, Contrib. Plasma Phys. 44, 508 (2004).

[10] Y. Oka et al., in Annual Report of NIFS, April 2005-Mar. 2006. 